\title{
A 3D Network of Graphene/Silicon/Graphene Sandwich Sheets as Anode for Li-Ion
}

\section{Battery}

Zhijun Feng ${ }^{1}$, Ang Fu ${ }^{1}$, Lun Chen ${ }^{1}$, Chenhui Huang ${ }^{1}$, Fei Pei ${ }^{1}$, Yanling $\mathrm{He}^{2}$, Xiaoliang Fang ${ }^{1}$, Baihua $\mathrm{Qu}^{1}$, Xinyi Chen ${ }^{1, *}$, Alan M. C. $\mathrm{Ng}^{2, *}$, Jingqin Cui ${ }^{1, *}$

${ }^{1}$ Pen-Tung Sah Institute of Micro-Nano Science and Technology, Xiamen University, Xiamen 361005, China

${ }^{2}$ Department of Physics, South University of Science and Technology, Shenzhen 518055, China

E-mails: chenxinyi@xmu.edu.cn, ngamc@ $\underline{\text { ngstc.edu.cn, jqcui@xmu.edu.cn }}$

ABSTRACT: A freestanding porous three-dimensional matrix composed of graphene/silicon/graphene sandwich sheets is proposed as anodes for lithium-ion batteries. Direct deposition of silicon on 3D-graphene secures active material attachment, improves conductivities while elastic graphene wrappings and numerous voids absorb Si volume expansions. With only $12 \mathrm{wt} \%$ of Si the design exhibits specific capacities of $0.62 \mathrm{mAh} \mathrm{cm}{ }^{-2}$ (423 $\mathrm{mAh} \mathrm{g}^{-1} \mathrm{Si}-\mathrm{C}$ composite) and long-term retentions of $\sim 60 \%$ at $0.7 \mathrm{~mA} \mathrm{~cm}^{-2}\left(0.45 \mathrm{~A} \mathrm{~g}^{-1}\right)$. At a higher current density $1.4 \mathrm{~A} \mathrm{~g} \mathrm{~g}^{-1}$, specific capacities stabilize at $70 \mathrm{mAh} \mathrm{g}^{-1}$ with Coulombic efficiencies (CE) higher than $99 \%$.

Keywords: 3D graphene; Sandwich structure; Silicon anode; Lithium-ion battery

\section{Introduction}

Lithium-ion battery (LIB) has been one dominant power source to liberate modern electronic devices' functions and portability [1]. Yet increasing desires for ultrathin, light devices demand novel redox chemistries and tailored architectures to dramatically improve LIB's energy density and longevity [2]. A promising anode material forming Li alloys, silicon however faces enormous volume changes resulting in crumbling and cracking, hence it requires extreme care in configuration designs [3]. Many slurry-casted electrodes with novel Si nanostructures have achieved excellent capacities over extended cycles [4]. Yet limited by cathode capacities and spaces in devices, in practical terms only a small amount of Si, usually accompanied by various forms of $\mathrm{C}$, is necessary to avoid unsafe swelling [5,6]. To make full 
usage of the active material, one efficient approach is applying conformal silicon over 3D current collectors, e.g. carbon foams that enhance the charge transfer and reduce the electrode mass [7].

Following these principles we employ 3D graphene (3DGr) as both the active material supporter and the current collector. Additionally, graphene has shown favorable futures as anodes, improved battery performances as wraps or slippery surfaces $[8,9,10,11]$. Through depositing Si films on 3DGr skeletons grown on Ni foams, a matrix is created with naturally interconnected active materials and increased surface areas, eliminating binders/conductive additives and shortening ionic diffusion pathways. A similar technique was proposed without dissolving the metal to maintain the mechanical strength [12]. Here $\mathrm{Ni}$ is removed and an additional graphene layer is coated over $\mathrm{Si}$, forming sandwich-type graphene/silicon/graphene sheets (referred as 3DGrSiGr hereafter, procedure summarized in Figure 1a). The matrix offers sufficient voids, limits agglomeration and increases conductivities, resulting in facilitated electrochemical reaction reversibility and enhanced cycling stabilities.
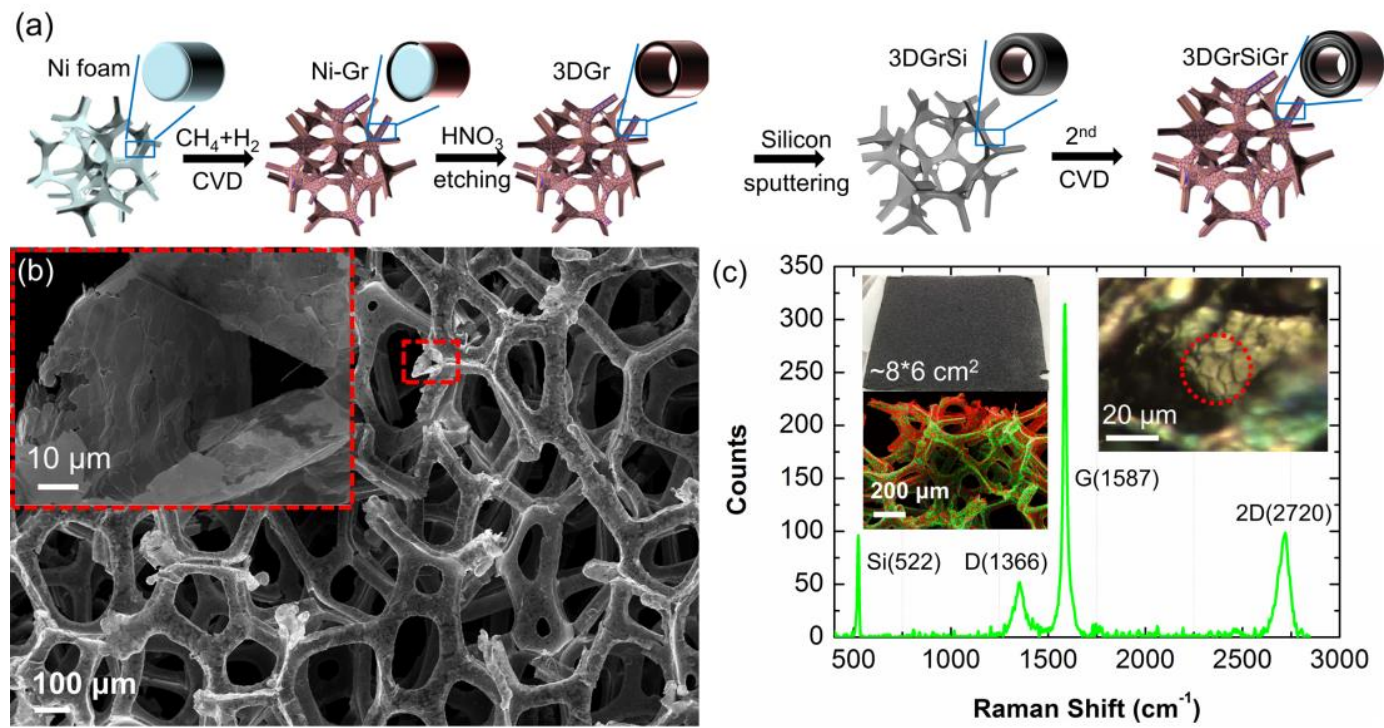

Figure 1. (a) Schematic 3DGrSiGr fabrication process; (b) SEM image of electrodes and the open end of an individual branch (Inset); (c) Raman spectrum of a 3DGrSiGr sheet (left top Inset). Left bottom Inset: EDS map indicates universally distributed C (red), Si (green). Right Inset: optical image of a branch surface with the laser spot indicated.

\section{Experimental}


3DGr was fabricated following the Ni-sacrificing method via chemical vapor deposition (CVD) [13]. Silicon films were deposited via angled magnetron sputtering followed by a second graphene CVD process. The prepared 3DGrSiGr (10-14 wt\% of Si) was then cut into circular disks of diameter $1.10 \mathrm{~cm}$ and of average weight $0.35 \pm 0.03 \mathrm{mg}$. Higher content of silicon can be achieved by simply pressing several layers graphene or precursor Ni foam together. Ni foam of any shape or size is commercially available and can be cold rolled into different thickness of various porosities. Therefore mass production of large electrode sheets is possible, averaging down the manufacturing cost. The disk anodes, several stacked together to remove the excessive voids, were assembled into CR2032 coin cells with lithium counter electrodes and Celgard 2400 separators. De-/charging were repeated at room temperature on a Neware BTS4000 testing system (Shenzhen, China) between 3.00-0.01 V at various current densities. Capacities were based on the total Si-C composite weight. More details are listed in Supporting Information.

\section{Results and discussion}

Scanning electron microscopy (SEM) images (Figure 1b) verify that $3 \mathrm{DGrSiGr}$ is immune from the sputtering or the CVD process. The inner empty spaces and the pores among interconnected hollow branches both supply sufficient buffer for stress rearranging and electrolyte diffusing while maintaining a much reduced mass. Compared to island-like depositions on copper foils or silicon wafers from the same process (Figure S1), Si films on 3DGr appear planar and nearly wrinkle-free, hinting conformal coverage of graphene and excellent attachment between $\mathrm{C}$ and $\mathrm{Si}$. The actual thickness of 400-nm deposition, observed from the film on the $\mathrm{Si}$ wafer, is $\sim 310 \mathrm{~nm}$, thinner than the diffusion limit of lithium in silicon[14]. In the Raman spectra (Figure 1c) characteristic G and 2D bands are from the outer-layer graphene covering the $\mathrm{Si}$ film which produces a crystalline-related transverse optical peak as the laser can penetrate only $\sim 100 \mathrm{~nm}$ into solid $[15,16]$. The EDS map reveals universal Si deposition over the graphene branches. One drawback of sputtering is that the 
film is usually deposited in a directional fashion and hence may not cover vertical surfaces evenly. However with the porous structure of 3DGr, many surfaces inside the network are able to be partially covered, creating non-continuous $\mathrm{Si}$ films that actually facilitate decreasing mechanical stresses.

The charge-discharge curves and cyclic voltammetry (CV) profiles (Figure 2) confirm both graphene and silicon contribute as active materials. During the $1^{\text {st }}$ lithiation process steep decreasing with a turn point near $0.70 \mathrm{~V}$, due to the formation of the solid electrolyte interface (SEI) layer, and a short stable period around $0.20 \mathrm{~V}$ from Li-Si alloying are observed. Below $0.15 \mathrm{~V}$ the potential decreases slowly with two quasi-plateaux near 0.12 and $0.08 \mathrm{~V}$ observed, corresponding to $\mathrm{Li}$ intercalation into graphene and the recrystallization of $\mathrm{Li}-\mathrm{Si}$ alloys, respectively $[17,18]$. The $1^{\text {st }}$ delithiation curve is obsereved with three flat regions located at $0.11,0.14 \mathrm{~V}$, related to the extraction of $\mathrm{Li}^{+}$from graphitic carbon, $0.23 \mathrm{~V}$ and one inconspicuous plateau at $0.41 \mathrm{~V}$, accociated with different stages of Li-Si dealloying [19, 20]. The following curves maintain similar smooth slopes, except that the SEI-formating turn point disappears, indicating reversible reactions. Accordingly, during the $1^{\text {st }} \mathrm{CV}$ cycle no detecable peak is observed in the cathodic scan due to the SEI formation. Along the anodic scan broad peaks between $0.2-0.6 \mathrm{~V}$ arose from $\mathrm{Li}$ extractions from $\mathrm{Si}$ and $\mathrm{C}$. Starting from the $2^{\text {nd }}$ cycle, a peak around $0.17 \mathrm{~V}$ with a shoulder $(\sim 0.20 \mathrm{~V})$ and another sharp peak near $0.02 \mathrm{~V}$ along the cathodic scan are recorded, coressponding to Li-C intercalation and Li-Si alloying [21]. Subsquently for the anodic scan, a bump splitting into two peaks, 0.22 and $0.29 \mathrm{~V}$, from $\mathrm{Li}$ extraction from graphene and transition of Li-Si crystalline alloys to amorphous alloys, and a weak broad peak around $0.50 \mathrm{~V}$, due to $\mathrm{Li}$ extracting from various $\mathrm{Li}-\mathrm{Si}$ compositions, are noticed [22]. The following cycles display almost identical patterns overlapping with each other, suggesting structurally stable electrodes undergoing reversible electrochemical reactions. For comparison, CV curves and charge-discharge profiles for 3DGr anodes are shown in Figure $\mathrm{S} 2$. 

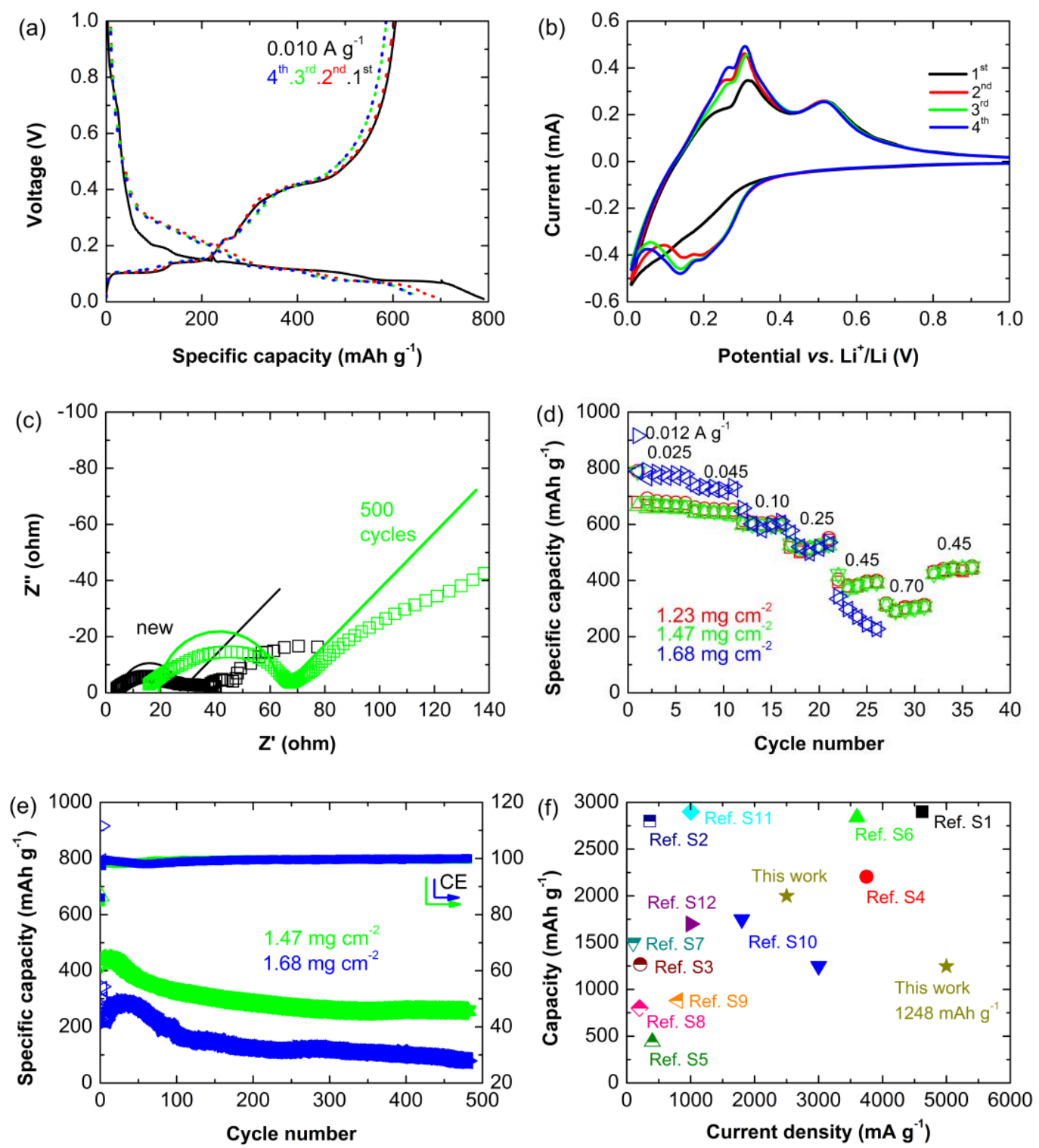

Figure 2. (a) Voltage-capacity profiles of $3 \mathrm{DGrSiGr}$ anode cycled at $0.010 \mathrm{~A} \mathrm{~g}^{-1}$ and (b) $\mathrm{CV}$ curves scanned at $0.1 \mathrm{mV} \mathrm{s}^{-1}$. Only data between $0.01-1.0 \mathrm{~V}$ are plotted; (c) Impedance measurements of an electrode before activation (black) and after 500 cycles (green) at $0.50 \mathrm{~A}$ $\mathrm{g}^{-1}$. The marks are experimental data and the lines are fitted data; (d) Rate performance at different current densities ( $\left.\mathrm{A} \mathrm{g}^{-1}\right)$ of varied mass loadings; (e) Cycling stability and CE of two electrodes at $0.50 \mathrm{~A} \mathrm{~g}^{-1}$. The electrodes are activated at $0.010 \mathrm{~A} \mathrm{~g}^{-1}$; (f) Comparison of electrochemical performances of our 3D structure with literature $\mathrm{Si}$-anode designs (all based on the Si mass).

The Nyquist plots (Figure 2c) of new cells and 500-cycle cells indicate stable electrode structures over the cycles. Before cycling the short-range interface resistance and chargetransfer impedance are 4.0 and $33.6 \Omega$, respectively. After de-/lithiation for 500 cycles at a charging density of $1.0 \mathrm{~mA} \mathrm{~cm}{ }^{-2}$, the resistances increase to 15.7 and $67.6 \Omega$, suggesting that structures have not changed dramatically. The considerably small values are results of much 
enhanced ionic diffusion with the pores in the network filled with electrolyte and Li ions. A Randles circuit including an interface resistance, a double layer capacitor, a charge-transfer impedance, and a Warburg impedance is assumed for the simulation. Galvanostatic performance of the 3DGrSiGr composite is assessed at various dis/-charge rates and the anodes show excellent capabilities and stabilities (Figure 2d). For three different loadings, initial specific capacities at the current density $0.025 \mathrm{~A} \mathrm{~g}^{-1}$ are all better than $700 \mathrm{mAh} \mathrm{g}^{-1}$, suggesting greatly enhanced power density. With mass loadings of 1.23 or $1.47 \mathrm{mg} \mathrm{Si}-\mathrm{C} \mathrm{cm}{ }^{-2}$, $650 \mathrm{mAh} \mathrm{g}^{-1}\left(\sim 0.60 \mathrm{mAh} \mathrm{cm}^{-2}\right)$ is achieved at $0.045 \mathrm{~A} \mathrm{~g}^{-1}$. The capacity decreases to 600,510 , and $390 \mathrm{mAh} \mathrm{g}^{-1}$ for $0.10,0.25$, and $0.45 \mathrm{~A} \mathrm{~g}^{-1}$, respectively. At $0.70 \mathrm{~A} \mathrm{~g}^{-1}$, a specific capacity $300 \mathrm{mAh} \mathrm{g}^{-1}$ is still obtained. For bulkier stacking, the ions need more time to reach far-end branches and lithiation becomes more irreversible, hence the specific capacity decreases rapidly to $200 \mathrm{mAh} \mathrm{g}^{-1}$. After 480 cycles at $0.7 \mathrm{~mA} \mathrm{~cm}^{-2}$ (Figure 2e), the specific capacity for $1.47 \mathrm{mg} \mathrm{cm}^{-2}$ stabilizes at $0.38 \mathrm{mAh} \mathrm{cm}^{-2}$, which is $\sim 61 \%$ of the first-cycle capacity. For 1.68 $\mathrm{mg} \mathrm{cm}{ }^{-2}$, the eventual capacity retention is only $20 \%$. Overall the 3DGrSiGr anodes are able to retain a capacity of magnitude $\sim 0.5 \mathrm{mAh} \mathrm{cm}^{-2}$, comparable to or higher than literature values while requiring much less active material [12,14,23]. A summary comparison with recent literature works is plotted in Figure $2 \mathrm{f}$ and listed in Table $\mathrm{S} 1$. At a high rate $1.0 \mathrm{~mA} \mathrm{~cm}-$ ${ }^{2}$, the capacity of $0.18 \mathrm{mAh} \mathrm{cm}^{-2}$ is achieved for a $3 \mathrm{DGrSiGr}$ anode ( $\mathrm{Gr}: \mathrm{Si}=88: 12$ ), while a 3DGr anode of same total mass reaches a capacity of $0.10 \mathrm{mAh} \mathrm{cm}$ (Figure S3). The porous matrix opens more accessibility for mass transport and active sites for electrochemical reactions, therefore holds higher capacities. Even at high current densities the CEs are above 98\% over 1000 cycles, indicating highly reversible reactions (Figure S4).

Composition variations at different phases of lithiation are examined with high-resolution Xray photoelectron spectroscopy (XPS) (Figure 3). Before cycling, a major $\mathrm{Si}_{2 \mathrm{p}}$ peak at 101.8 $\mathrm{eV}$ (Si-C), a sharp peak near $284.5 \mathrm{eV}$ associated with $\mathrm{C} \mathrm{sp}{ }^{2}$ and a weak $\mathrm{C}=\mathrm{O}$ peak at 288.5 $\mathrm{eV}$ are detected. The $\mathrm{O}_{1 \mathrm{~s}}$ peaks could have come from the $\mathrm{Si}-\mathrm{O}$ bond $(532.9 \mathrm{eV})$ and 
structures with $\mathrm{C}=\mathrm{O}, \mathrm{O}-\mathrm{SiC}$ bonds $(531.5 \mathrm{eV})$. After full lithiation (discharged to $0.01 \mathrm{~V}$ ), clear $\mathrm{Li}_{1 \mathrm{~s}}$ spectrum of two sub-peaks at 53.4 and $54.6 \mathrm{eV}$ appears. Possible chemical structures include $\mathrm{Li}-\mathrm{OH}$ and $\mathrm{Li}_{\mathrm{x}} \mathrm{SiO}_{\mathrm{y}}$ [24]. The $\mathrm{Si}_{2 \mathrm{p}}$ signal confirms the formation of $\mathrm{Li}_{\mathrm{x}} \mathrm{SiO}_{\mathrm{y}}$ with the $102.9 \mathrm{eV}$ peak. Three additional sub-peaks are observed at positions 99.5, 100.5 for possible $\mathrm{Si}-\mathrm{Si}$ and $\mathrm{Li}-\mathrm{Si}$, and 101.7 for $\mathrm{Si}-\mathrm{C}$, respectively. In the $\mathrm{C}_{1 \mathrm{~s}}$ spectrum, the broadened C-C bond slightly shifts to $284.1 \mathrm{eV}$ while the wide 289.1 peak is possibly the combination of $\mathrm{C}=\mathrm{O}$ and C-Li. Two additional peaks appear at $285.9 \mathrm{eV}$ from PEO-type electrolytes, and $282.4 \mathrm{eV}$ from $\mathrm{Si}-\mathrm{C}$ bonding. For $\mathrm{O}_{1 \mathrm{~s}}, \mathrm{Si}-\mathrm{O}$ and $\mathrm{Li}_{\mathrm{x}} \mathrm{SiO}_{\mathrm{y}}$ are formed as a main peak $530.9 \mathrm{eV}$ with a shoulder at $532.5 \mathrm{eV}$ is observed. The spectra again confirm that $\mathrm{Si}$ and graphene both contribute to hosting lithium and stay attached during cycling. XPS results of 3DGr-only anodes at the lithiation status are shown in Figure S5.
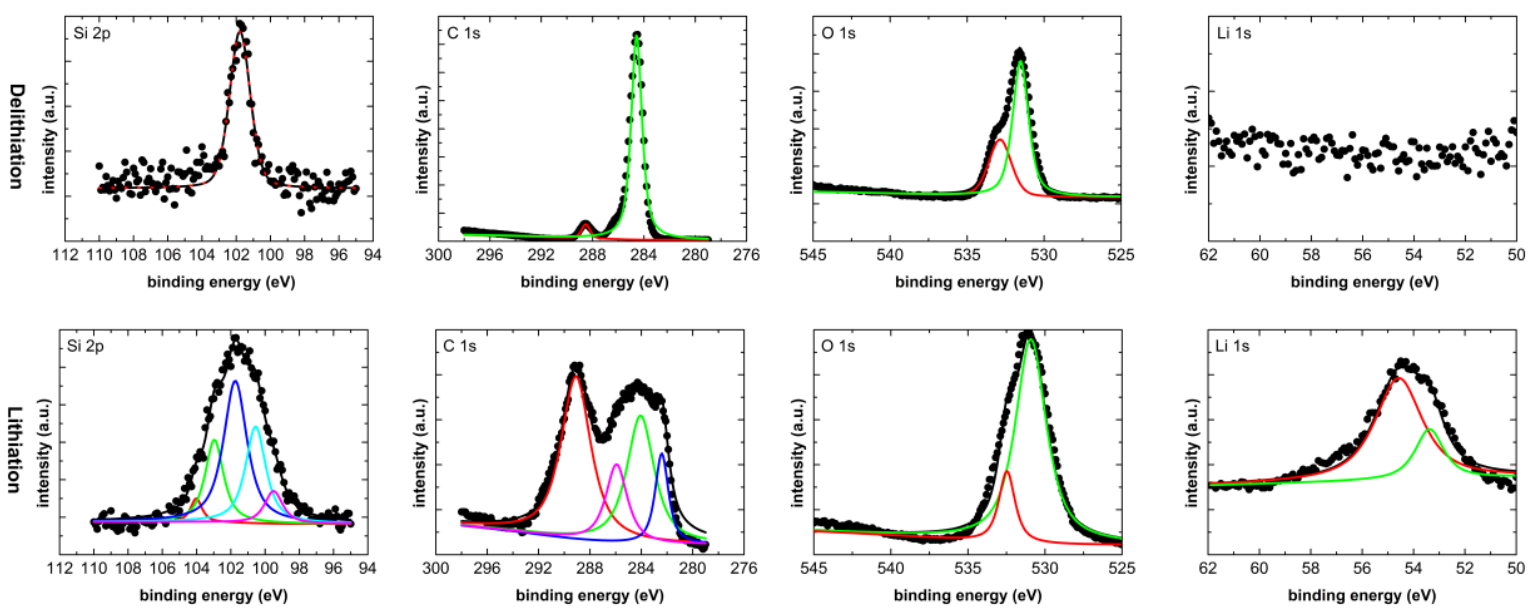

Figure 3. XPS spectra of 3DGrSiGr at de-lithiation status and lithiation status.

Structurally the 3DGrSiGr remains its soft, flexible nature after many cycles. Upon rinsing with solvent a full-shaped anode can be freed from the rest cell parts. However morphology evolutions at the micro-scale are clearly identified with SEM (Figure 4). The anode having experienced only one complete cycle looks much similar to the pristine network in a way that belts and voids are obviously distinguishable. Yet after 500 cycles, empty spaces between branches disappear and twisted surfaces with wrinkles appear here and there in a random fashion. The wrinkled areas are dominantly $\mathrm{Si}$ with the rest area containing both $\mathrm{Si}$ and $\mathrm{C}$ 
(Figure S6). The initial branches/tubes have transformed into flat belts from stacking and battery assembling. Then during cycling belts, composed of ductile graphene and silicon films, expand horizontally into the empty space while the voids absorb the volume expansion and rearrange the belts into wrinkles. Aside from wrinkles flat surfaces with mud-cracks of various widths are seen. Higher magnification images show that for 1-cycle anode, shallow cracking lines are just starting to appear on the Si film, while after 500 cycles silicon has evolved into numerous nano-pillars. This transition greatly enhances interactions between the anode and electrolyte, and explains why specific capacities drop first but increase later during extended cycling. Meanwhile Si films are shown continuously anchored to the 3DGr skeleton during cycling (Figure S6), proving efficient structural perseverance.

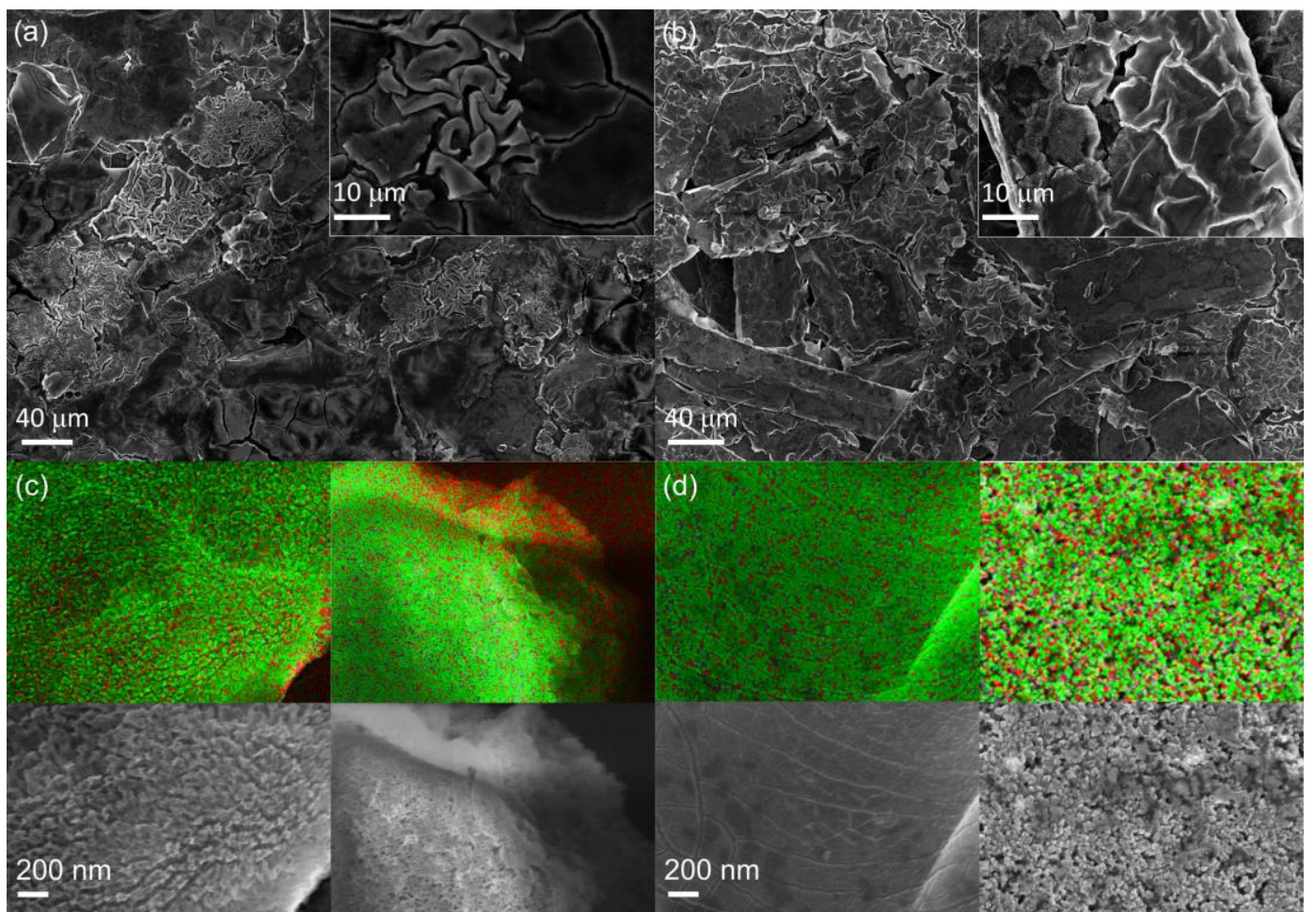

Figure 4. SEM images of 3DGrSiGr electrodes after (a) 500 cycles and (b) only 1 de/lithiation cycle. The insets display striking morphology contrasts between wrinkled regions and cracked regions. The morphology and element distribution of wrinkles (left) and cracks (right) after 500 cycles (c), and after only 1 cycle (d). EDS maps on the top show abundant Si (green) and dense $\mathrm{C}$ (red) overlapping in all morphologies.

The experimental results agree well with the hypothesis that volume change-induced mechanical stress and material pulverization is one core reason of performance decaying. Among the samples thinner Si deposition performs better as a thinner volume creates less 
concentrated local stress hence is less likely to fall off the skeleton. The strong graphenesilicon adhesion effectively prevents nanostructure agglomeration or loss of active material, both profitable for battery performances. More important, the few-layer graphene can rearrange its stacking easily following the Si film distorting as inter-layer sliding and interfacial sliding face ultralow frication at the atomic scale [11,25]. Hence with only a negligible volume and $\sim 10 \%$ mass added, the anode realizes an $80 \%$ increase in areal capacities.

\section{Conclusions}

In summary, a 3D network of sandwich sheets with silicon thin film wrapped between graphene is proposed as free-standing anodes. The protected $\mathrm{Si}$ experiences limited and stable formation of SEI, while the 3DGr skeleton maintains the conductive pathway. As predicted, an additional top layer graphene protection helps improving electrochemical stability [10,11]. With only $12 \mathrm{wt} \%$ of $\mathrm{Si}$, maximum specific capacities tested at 0.025 and $0.45 \mathrm{~A} \mathrm{~g}^{-1}$ reach 680, $420 \mathrm{mAh} \mathrm{g}^{-1}$, corresponding to 1.0 and $0.62 \mathrm{mAh} \mathrm{cm}^{-2}$, respectively. After 500 cycles under $0.45 \mathrm{~A} \mathrm{~g} \mathrm{~g}^{-1}$ the structure achieves decent capacity retentions over $60 \%$. This novel combination of 3D architecture and sandwich wrapping significantly reduces the loss of active material-current collector contact, grants sufficient room for volume expansion, and is applicable to many materials. Being inherently superlight and highly flexible, 3DGrSiGr poses excellent potentials for applications in portable and wearable devices.

\section{Supporting information}

Additional information on electrode morphologies, electrochemical performances.

\section{Acknowledgements}

The authors thank NSF of Fujian Province, China (No. 2017J01103) and the Graphene Project of Fujian Provincial Development and Reform Commission. The project is also funded by NSFC (Nos. 21303143, 11574255). Ms. Lingling Zheng is sincerely appreciated for her support with material characterizations.

Declarations of interest: none.

\section{References}


[1] J. B. Goodenough, Electrochemical Energy Storage in a Sustainable Modern Society. Energy Environ. Sci. 2014, 7, 14-18.

[2] J. W. Choi, D. Aurbach, Promise and Reality of Post-Lithium-Ion Batteries with High Energy Densities. Nature Reviews Materials 2016, 1, 16013.

[3] U. Kasavajjula, C. Wang, A. J. Appleby, Nano- and Bulk-Silicon-Based Insertion Anodes for Lithium-Ion Secondary Cells. J. Power Sources 2007, 163, 1003-1039.

[4] Y. Liu, G. Zhou, K. Liu, Y. Cui, Design of Complex Nanomaterials for Energy Storage: Past Success and Future Opportunity. Acc. Chem. Res., 2017, 50 , 2895-2905.

[5] R. Dash, S. Pannala, Theoretical Limits of Energy Density in Silicon-Carbon Composite Anode Based Lithium Ion Batteries. Sci. Reports. 2016, 6, 27449.

[6] X. Shen, Z. Tian, R. Fan, L. Shao, D. Zhang, G. Cao, L. Kou,Y. Bai, Research Progress on Silicon/Carbon Composite Anode Materials for Lithium-Ion Battery. J. Energy Chem. 2018, 27, 1067-1090.

[7] Y. Yue, H. Liang, 3D Current Collectors for Lithium-Ion Batteries: A Topical Review, Small Methods 2018, 2, 1800056.

[8] K. Chen, Q. Wang, Z. Niu, J. Chen, Graphene-Based Materials for Flxible Energy Storage Devices. J. Energy Chem. 2018, 27, 12-24.

[9] R. Raccichini, A. Varzi, D. Wei, S. Passerini, Critical Insight into the Relentless Progression toward Graphene and Graphene-Containing Materials for Lithium-Ion Battery Anodes. Adv. Mater. 2017, 29, 1603421.

[10] S. Suresh, Z. P. Wu, S. F. Bartolucci, S. Basu, R. Mukherjee, T. Gupta, P. Hundekar, Y. Shi, T.-M. Lu, N. Koratkar, Protecting Silicon Film Anodes in Lithium-Ion Batteries Using an Atomically Thin Graphene Drape. ACS Nano 2017, 11, 5051-5061.

[11] S. Basu, S. Suresh, K. Ghatak, S. F. Bartolucci, T. Gupta, P. Hundekar, R. Kumar, T.-M. Lu, D. Datta, Y. Shi, N. Koratkar, Utilizing van der Waals Slippery Interfaces to Enhance the 
Electrochemical Stability of Silicon Film Anodes in Lithium-Ion Batteries. ACS Appl. Mater. Interfaces 2018, 10, 13442-13451.

[12] A. Mukanova, A. Nurpeissova, A. Urazbayev, S.-S. Kim, M. Myronov, Z. Bakenov, Silicon Thin Film on Graphene Coated Nickel Foam as an Anode for Li-Ion Batteries. Electrochimica Acta 2017, 258, 800-806.

[13] Z. Chen, W. Ren, L. Gao, B. Liu, S. Pei, H.-M. Cheng, Three-Dimensional Flexible and Conductive Interconnected Graphene Networks Grown by Chemical Vapour Deposition. Nature Materials 2011, 10, 424-428.

[14] F. Farmakis, C. Elmasides, P. Fanz, M. Hagen, N. Georgoulas, High energy density amorphous silicon anodes for lithium ion batteries deposited by DC sputtering. J. Power Sources, 2015, 293, 301-305.

[15] Z. Iqbal, S. Veprek, Raman Scattering from Hydrogenated Microcrystalline and Amorphous Silicon. J. Phys. C: Solid State Phys. 1982, 15, 377-392.

[16] A. C. Ferrari, J. C. Meyer, V. Scardaci, C. Casiraghi, M. Lazzeri, F. Mauri, S. Piscanec, D. Jiang, K. S. Novoselov, S. Roth, and A. K. Geim, Raman Spectrum of Graphene and Graphene Layers. Phys. Rev. Lett. 2006, 97, 187401.

[17] M. K. Datta, P. N. Kumta, In situ Electrochemical Synthesis of Lithiated Silicon-Carbon based Composites Anode Materials for Lithium Ion Batteries. J. Power Sources 2009, 194, 1043-1052.

[18] L. Ji, H. Zheng, A. Ismach, Z. Tan, S. Xun, E. Lin, V. Battaglia, V. Srinivasan, Y. Zhang, Graphene/Si Multilayer Structure Anodes for Advanced Half and Full Lithium-Ion Cells. Nano Energy 2012, 1, 164-171.

[ 19 ] H. Guo, D. Long, Z. Zheng, X. Chen, A. M. C. Ng, M. Lu, Defect-Enhanced Performance of a 3D Graphene Anode in a Lithium-Ion Battery. Nanotechnology 2017, 28, 505402. 
[20] A. R. Jiménez, R. Klöpsch, R. Wagner, U. C. Rodehorst, M. Kolek, R. Nölle, M. Winter, T. Placke, A Step toward High-Energy Silicon-Based Thin Film Lithium Ion Batteries. ACS Nano 2017, 11, 4731-4744.

[ 21 ] M. Choi, J.-C. Kim, D.-W. Kim, Waste Windshield-Derived Silicon/Carbon Nanocomposites as High-Performance Lithium-Ion Battery Anodes. Sci. Reports 2018, 8, 960. [22] Y. Yao, M. T. McDowell, I. Ryu, H. Wu, N. Liu, L. Hu. W. D. Nix, Y. Cui, Interconnected Silicon Hollow Nanospheres for Lithium-Ion Battery Anodes with Long Cycle Life. Nano Lett. 2011, 11, 2949-2954.

[23] M. Ashuri, Q. He, L. L. Shaw, Silicon as a Potential Anode Material for Li-Ion Batteries: Where Size, Geometry and Structure Matter. Nanoscale 2016, 8, 74-103.

[24] B. Philippe, R. Dedryvère, J. Allouche, F. Lindgren, M. Gorgoi, H. Rensmo, D. Gonbeau, K. Edström, Nanosilicon Electrodes for Lithium-Ion Batteries: Interfacial Mechanisms Studied by Hard and Soft X-ray Photoelectron Spectroscopy. Chem. Mater. 2012, 24, $1107-$ 1115

[25] H. J. Park, J. Meyer, S. Roth, V. Skákalová, Growth and Properties of Few-Layer Graphene Prepared by Chemical Vapor Deposition. Carbon 2010, 48, 1088-1094. 